\title{
Le Rapport de la Commission Tremblay (1953-1956), testament politique de la pensée traditionaliste canadienne-française
}

\section{Dominique Foisy-Geoffroy}

Volume 60, numéro 3, hiver 2007

URI : https://id.erudit.org/iderudit/015960ar

DOI : https://doi.org/10.7202/015960ar

Aller au sommaire du numéro

Éditeur(s)

Institut d'histoire de l'Amérique française

ISSN

0035-2357 (imprimé)

1492-1383 (numérique)

Découvrir la revue

Citer cet article

Foisy-Geoffroy, D. (2007). Le Rapport de la Commission Tremblay (1953-1956), testament politique de la pensée traditionaliste canadienne-française. Revue d'histoire de l'Amérique française, 60(3), 257-294.

https://doi.org/10.7202/015960ar
Résumé de l'article

En 1953, le premier ministre Maurice Duplessis confie à la Commission Tremblay le mandat d'étudier le problème des relations fédérales-provinciales au Canada du point de vue fiscal, dans le cadre de la lutte menée par Québec contre la politique centralisatrice d'après-guerre du gouvernement fédéral. Les commissaires, qui déposent leur rapport en 1956, fondent leurs recommandations sur un impressionnant étaiement philosophique, auquel cet article est consacré. Sous l'impulsion de trois des intellectuels canadiens-français les plus en vue de l'époque, Esdras Minville, le père Richard Arès, s.j. et François-Albert Angers, la commission a en effet produit une véritable somme théorique et pratique du nationalisme traditionaliste canadien-français, synthétisant réflexions et projets de réforme qui mûrissaient depuis les années 1920. Nous nous penchons ici plus précisément sur la pensée politique " classique-chrétienne " inspirant le rapport, qui justifie philosophiquement l'autonomisme canadien-français, donne leur sens aux exhaustives recommandations du rapport et fonde l'ambitieux projet de civilisation nationaliste, conservateur et catholique qu'élaborent les commissaires autour du concept de " fédéralisme social ".
Tous droits réservés @ Institut d'histoire de l'Amérique française, 2007
Ce document est protégé par la loi sur le droit d'auteur. L’utilisation des services d'Érudit (y compris la reproduction) est assujettie à sa politique d'utilisation que vous pouvez consulter en ligne.

https://apropos.erudit.org/fr/usagers/politique-dutilisation/ 


\title{
Le Rapport de la Commission Tremblay (1953-1956), testament politique de la pensée traditionaliste canadienne-française ${ }^{\mathrm{I}}$
}

\author{
DOMINIQUe FoISY-GEOFFroY \\ Département d'histoire \\ Université Laval
}

RÉSUMÉ • En 1953, le premier ministre Maurice Duplessis confie à la Commission Tremblay le mandat d'étudier le problème des relations fédérales-provinciales au Canada du point de vue fiscal, dans le cadre de la lutte menée par Québec contre la politique centralisatrice d'après-guerre du gouvernement fédéral. Les commissaires, qui déposent leur rapport en 1956, fondent leurs recommandations sur un impressionnant étaiement philosophique, auquel cet article est consacré. Sous l'impulsion de trois des intellectuels canadiens-français les plus en vue de l'époque, Esdras Minville, le père Richard Arès, s.j. et François-Albert Angers, la commission a en effet produit une véritable somme théorique et pratique du nationalisme traditionaliste canadien-français, synthétisant réflexions et projets de réforme qui mûrissaient depuis les années 1920. Nous nous penchons ici plus précisément sur la pensée politique «classique-chrétienne» inspirant le rapport, qui justifie philosophiquement l'autonomisme canadien-français, donne leur sens aux exhaustives recommandations du

1. Cet article a pu être rédigé grâce à une bourse de doctorat du CRSH et du FQRSC. Nous aimerions remercier Sylvie Lacombe, Jocelyn Létourneau, Martin Pâquet et Pierre Trépanier ainsi que les évaluateurs anonymes de la Revue d'histoire de l'Amérique française pour leur aide et leurs commentaires. Nos remerciements également à Michèle $S$. Jean qui a bien aimablement accepté de nous laisser consulter la transcription des entrevues qu'elle et René Durocher ont menées avec certains membres de la Commission Tremblay en 1971. "Traditionaliste» fait ici référence à l'idéologie antimoderne née en réaction à la Révolution française, et non au mouvement catholique d'opposition à la réforme liturgique proposée par le concile de Vatican II. Il est dans notre esprit synonyme de "conservateur» (au sens idéologique) et de "clérico-nationaliste», concept propre au Québec que nous dépouillons cependant de son sens péjoratif originel. 
rapport et fonde l'ambitieux projet de civilisation nationaliste, conservateur et catholique qu'élaborent les commissaires autour du concept de «fédéralisme social».

ABSTRACT • In 1953, Quebec premier Maurice Duplessis mandated the Tremblay Commission to inquire into the fiscal aspects of federal-provincial relations in Canada, in the context of the province's struggle against the federal government's postwar centralizing policies. The commission's report, published in 1956, based its recommendations on wideranging philosophical reflections, which this article seeks to explore. Under the aegis of three of the most prominent French-Canadian intellectuals of the time, Esdras Minville, Richard Arès, S.J. and François-Albert Angers, the commissioners produced a masterful survey, both theoretical and practical, of traditionalist French-Canadian nationalism, synthesizing both reflections and reform projects that had been developing since the 1920s. The article focuses in particular on the report's «classical-Christian» political thought, which provided the philosophical underpinning for French-Canadian autonomism, justified the report's exhaustive recommendations and was the basis for the ambitious civilizing project, nationalist, conservative and Catholic, that the commissioners developed through the notion of «social federalism ».

\section{L} e 12 février 1953, le lieutenant-gouverneur de la province de Québec sanctionne la Loi instituant une commission royale d'enquête sur les problèmes constitutionnels, proposée par le gouvernement Duplessis. La Commission Tremblay, comme on allait la nommer, est lancée et allait déposer son rapport trois ans plus tard, en février $1956^{2}$. Fruit de l'activisme de la Chambre de commerce de Montréal, à l'époque très engagée dans les débats de société, la commission a pour mandat d' "enquêter sur les problèmes constitutionnels " et d'étudier le problème de la répartition des impôts au sein de l'État canadien. L'équipe de commissaires, sous la

2. Les études sur la Commission Tremblay se résument à quelques articles et thèses de maîtrise: René Durocher et Michèle S. Jean, «Duplessis et la Commission royale d'enquête sur les problèmes constitutionnels, 1953-1956", Revue d'histoire de l'Amérique française (RHAF), 25,3 (décembre 1971):337-363; Marc Chevrier, «La conception pluraliste et subsidiaire de l'État dans le rapport Tremblay de 1956: entre l'utopie et la clairvoyance ", Cahiers d'histoire du Québec au xx siècle, 2 (été 1994): 45-57; Martin Pâquet, «Des lettres mortes... La Commission Tremblay et l'immigration (1953-1956)", Revue d'études canadiennes, 30,4 (hiver 1995-1996): 52-74; David Kwavnick, "Editor's Introduction», The Tremblay Report (Toronto, McClelland and Stewart Limited, coll. «Carleton Library », 1973), vii-xix; René Gagnon, Étude du fédéralisme dans le Rapport Tremblay, essai de maîtrise (science politique), Université Laval, 1961 et Les sources du Rapport Tremblay en ce qui concerne les relations fédérales-provinciales, essai de maîtrise (science politique), Université Laval, 1961 ; Richard Arès, «Le commissaire royal», L'Action nationale, 65,9-10 (mai-juin 1976) : 689-705 [sur Esdras Minville]; Gaétan Legault, «L'État selon le rapport de la Commission Tremblay ", Tradition et progrès, 4,1 (hiver 1961 [1957]) : 20-27. 
présidence du juge Thomas Tremblay, est composée du père Richard Arès, s.j., de Paul-Henri Guimont, d'Esdras Minville, d'Honoré Parent et de John P. Rowat ${ }^{3}$.

En politique canadienne, les années 1940 à 1960 sont marquées par une forte poussée centralisatrice de la part du gouvernement fédéral. La montée d'un nationalisme proprement canadien consécutive au relâchement du lien avec la Grande-Bretagne et face à l'influence croissante des États-Unis sur tous les aspects de la vie canadienne amène de nombreux Canadiens anglais à vouloir affirmer le caractère de gouvernement national du gouvernement fédéral et à combattre les forces centrifuges qui y font obstacle, assez fortes voire dominantes au Canada jusqu'aux années 1930. Parallèlement, l'intensité de la crise économique des années 1930 pousse les municipalités et les provinces au bord du gouffre. Pressé d'agir, le gouvernement fédéral, sous l'impulsion de la haute fonction publique, élabore une politique inspirée des théories économiques keynésiennes. Selon celles-ci, l’État doit employer ses leviers de contrôle économique en temps de crise pour relancer une économie atrophiée: contrôle de la monnaie par la banque centrale, investissements publics, développement de la sécurité sociale comme outil économique, etc. Cette politique est appuyée sur le plan théorique par le rapport de la Commission Rowell-Sirois sur les relations entre le Dominion et les provinces (19371940). Pour exercer un contrôle efficace de l'économie et ainsi atténuer l'effet des cycles économiques sur la population, Ottawa sent le besoin de centraliser tous les pouvoirs qui peuvent y contribuer, y compris en matière de sécurité sociale. Par ailleurs, l'offensive centralisatrice ne se confine pas au domaine fiscal et touche aussi le domaine social et le domaine culturel, sous l'impulsion du rapport de la Commission Massey (1949-1951). Ainsi, la table est mise pour un formidable bras de fer politique et juridique entre les provinces et le gouvernement fédéral. C'est à ce mouvement de fond qu'entend répondre la Commission Tremblay, au nom du Québec - et du Canada français.

Les recommandations contenues dans le rapport sont très exhaustives et ne laissent à peu près aucun domaine en plan, de la culture à l'agriculture, de l'exploitation des ressources naturelles à la santé et à la sécurité sociale. En général, elles tendent à valoriser sans intransigeance la décentralisation et la préservation de la responsabilité des individus, le contrôle

3. Thomas Tremblay, dir., Rapport de la Commission royale d'enquête sur les problèmes constitutionnels [ci-après Rapport Tremblay] ([Québec], Province de Québec, 1956), 1 : ix-xi, xv. 
vigilant de l'État sur l'exploitation des ressources naturelles, une certaine planification de l'économie et un soutien actif mais prudent de la culture canadienne-française par l'État québécois. Les principales recommandations fiscales sont quant à elles radicales: les commissaires proposent en gros d'accorder l'exclusivité du champ de l'impôt indirect au gouvernement fédéral (taxes de vente, droits de douane) et l'exclusivité du champ de l'impôt direct aux gouvernements provinciaux - ou formule «100-100100 ", c'est-à-dire que $100 \%$ de l'impôt sur le revenu des particuliers, $100 \%$ de l'impôt sur le revenu des entreprises et $100 \%$ de l'impôt sur les successions serait attribué aux provinces, qui disposeraient ainsi des ressources financières les plus importantes. Cela s'accompagnerait d'une remise complète de l'administration des programmes de sécurité sociale aux provinces ainsi que d'une formule de péréquation pour soutenir les plus pauvres d'entre elles. L'objectif, en dernière analyse, est de rendre à chaque gouvernement les sources de financement lui permettant d'assumer les responsabilités qui lui ont été attribuées par l'Acte de l'Amérique du Nord britannique ${ }^{4}$.

Après avoir reçu un enterrement de première classe à la suite de son dépôt, épisode relaté avec brio par René Durocher et Michèle S. Jean ${ }^{5}$, le Rapport Tremblay est finalement réhabilité par Jean Lesage lors de la campagne électorale provinciale de 1960. Selon les paroles mêmes du premier ministre Robert Bourassa en 1971, c'est en fait tout l'effort constitutionnel québécois des années 1960 qui se serait inscrit dans la foulée des recommandations et des "grandes orientations» du Rapport Tremblay ${ }^{6}$. Les gouvernements québécois de la Révolution tranquille ont pour ce faire également bénéficié de la brèche pratiquée dans le régime fiscal canadien de l'après-guerre par l'adoption, en 1954, d'une loi permettant au gouvernement du Québec de prélever un impôt sur le revenu des particuliers de $15 \%$, ramené à $10 \%$ après entente avec le gouvernement fédéral. Jean et Durocher ont bien établi le rôle important, déclencheur, joué par le mouvement d'opinion créé par les audiences de la commission ainsi que par les interventions personnelles du juge Tremblay auprès du premier ministre Duplessis pour vaincre les réticences de ce dernier à lever cet impôt ${ }^{7}$.

4. Ibid., 3-II : 236-249.

5. R. Durocher et M. Jean, "Duplessis et la Commission royale d'enquête...», loc. cit., 358362.

6. Cité par Richard Arès, Nos grandes options politiques et constitutionnelles (Montréal, Éditions Bellarmin, 1972), 51.

7. R. Durocher et M. Jean, «Duplessis et la Commission royale d'enquête... », loc. cit., 346350 . 
Mais le Rapport Tremblay est bien plus qu'un simple épisode de l'éternelle saga des relations fédérales-provinciales au Canada. Il diffère de la plupart des rapports de commission d'enquête par le souci des commissaires de dépasser la simple analyse sur le plan technique du problème qui fait l'objet de l'enquête pour s'élever à l'étude exhaustive de ses tenants et aboutissants philosophiques, moraux et historiques. Il fait ainsi figure de véritable somme théorique et pratique du nationalisme traditionaliste canadien-français et devient du coup un exceptionnel sujet d'étude d'histoire intellectuelle. Au travers des analyses historiques et des propositions de réforme, nous trouvons en effet dans le Rapport Tremblay l'exposé le plus synthétique de la pensée politique canadienne-française d'inspiration traditionaliste (ou clérico-nationaliste) du $\mathrm{xx}^{\mathrm{e}}$ siècle. C'est cette pensée politique, l'âme du rapport, plutôt oubliée de nos jours, que nous remettons en lumière dans cet article. Elle nous conduit au cœur de l'un des problèmes fondamentaux de la politique, soit celui des rapports entre politique et transcendance. À l'origine de ce problème, un choix primordial: ou Dieu existe, ou il n'existe pas. Les conséquences de ce choix, de cet acte de foi, sont immenses dans tous les domaines de la vie humaine. En politique, lieu où l'être humain donne une orientation rationnelle aux destinées de la société, ce choix a évidemment une grande portée. En effet, si Dieu existe, si le monde et l'homme sont sa création, ordonnés à une fin déterminée qu'ils doivent atteindre par divers moyens, la politique doit se conformer à cet ordre qui dépasse l'homme et lui fixe des balises. À l'inverse, si Dieu n'existe pas, l'homme est seul maître de son destin et la politique devient non pas moyen d'accomplir une fin déterminée par une entité supérieure, mais outil prométhéen permettant à l'homme de s'arracher à sa condition et de devenir à lui-même son propre dieu, de créer de toutes pièces un nouvel homme, une nouvelle société, une nouvelle morale, de nouvelles règles régissant les rapports entre les personnes. C’est la portée, symbolique sans doute, mais néanmoins révélatrice d'un enjeu de première importance, de la mention de Dieu en tête des constitutions ainsi que de la présence de signes religieux, tel un crucifix, dans les chambres d'assemblée. Bien ancrée dans la réalité canadienne-française comme il se doit pour une ouvre traditionaliste, c'est en définitive sur ce questionnement universel et fondamental, dont l'actualité nous rappelle la permanence presque quotidiennement, que le Rapport Tremblay débouche. 


\section{I - LA PERSONNALITÉ INTELLECTUELLE DU RAPPORT TREMBLAY}

Si le Rapport Tremblay a une si grande valeur pour l'histoire des idées, cela est principalement dû à l'apport de trois intellectuels de premier plan liés à la commission, soit Esdras Minville, Richard Arès et François-Albert Angers, intellectuels qui sont sans doute les plus brillants (et fidèles...) disciples de Lionel Groulx et qui comptent parmi les plus fermes tenants du traditionalisme canadien-français. Il faut en effet bien comprendre que le Rapport Tremblay, du point de vue intellectuel, n'est pas tant un virage annonçant la modernité québécoise qu'un aboutissement, le résultat de décennies de réflexion et de débats sur tous les aspects de la vie canadienne-française menée dans les milieux nationalistes traditionalistes. Dans le Rapport Tremblay, on a repris et mis à jour la synthèse catholique et nationaliste qu'on a vu cheminer depuis des décennies dans la revue L'Action française et dans son héritière, L'Action nationale, dans le quotidien Le Devoir, dans L'Actualité économique, dans les Semaines sociales du Canada, les brochures de l'École sociale populaire et la revue Relations animées par les dynamiques pères jésuites, dans le Programme de restauration sociale de 1933, dans le programme politique de l'Action libérale nationale, puis dans celui du Bloc populaire canadien, dans les œuvres des Groulx, Arès, Minville, Angers et combien d'autres. Dans l'ensemble, les réformes contenues dans le Rapport Tremblay sont issues de la dynamique propre du nationalisme traditionaliste canadien-français depuis au moins une trentaine d'années. Leur vigueur ne nous paraît pas attribuable à une sorte d'effet d'entraînement causé par le jeune mouvement néonationaliste ${ }^{8}$, pas plus que le rapport ne constitue la «Bible du néo-nationalisme ${ }^{9}$. Une telle méprise ne peut s'expliquer que par la méconnaissance de l'histoire du traditionalisme canadien-français.

Le commissaire Esdras Minville, alors dans la cinquantaine avancée, est arrivé à l'âge des grandes œuvres. C'est lui qui, d'après Richard Arès, est la véritable autorité intellectuelle de la Commission Tremblay et qui préside à ce grand travail de synthèse. Toujours selon Arès, c'est Minville qui,

8. Michael Behiels, Prelude to Quebec's Quiet Revolution: Liberalism versus Neo-Nationalism, 19451960 (Montréal, McGill-Queen's University Press, 1985), 212. Il semble toutefois que l'analyse de Behiels s'avère plus juste si on l'applique à l'évolution de la droite canadienne-française à partir de la fin des années 1950, qui devient de plus en plus étatiste et délaisse ses traditionnelles réticences doctrinales face au principe des nationalités, notamment. Voir à ce sujet Xavier Gélinas, La droite intellectuelle québécoise et la Révolution tranquille (1956-1966), thèse de doctorat (histoire), York University, 2001, 364-462.

9. Louis Balthazar, Bilan du nationalisme au Québec (Montréal, Éditions de l’Hexagone, 1990), 119. 
après s'être plongé dans les travaux et mémoires soumis à la commission, aurait tracé les grandes lignes des recommandations du rapport, qui porteraient presque toutes sa marque ${ }^{10}$. C'est cependant la section intitulée «La province de Québec et le cas canadien-français» qui constitue sa contribution la plus importante au Rapport Tremblay. Ce texte d'une très grande densité, dans lequel Minville a concentré les conclusions essentielles de toute une vie de recherche et de réflexion, est en effet le cœur du rapport. Il y formule les raisons les plus profondes des revendications autonomistes québécoises en tentant de définir ce qu'est être canadienfrançais.

Posons tout d'abord que Minville, comme tout le rapport d'ailleurs, adopte une perspective traditionaliste et antimoderne sur ce qu'est le monde, l'homme et la vie. Entendons-nous: il ne rejette pas toute forme de modernisation économique ou politique, souhaitant plutôt leur harnachement à sa conception du monde traditionaliste. C'est le cour de la modernité qu'il rejette, la modernité philosophique (au sens pur) et ses caractères d'autoréférentialité, d'autofondation et d'autolégitimation, en somme son caractère anthropocentrique et sa tendance corollaire à rejeter l'idée d'un ordre supérieur à la volonté humaine et de normes transcendantes et absolues au profit de la liberté de chacun de déterminer ce qui fait son bonheur et quelles sont les voies pour y parvenir ${ }^{11}$. L'extrait suivant ne laisse guère d'équivoque à ce sujet:

L'ordre de la vie procède ainsi de normes transcendantes, permanentes et universelles, donc valables pour tous les hommes et à toutes les époques et dans tous les milieux. [...] Qu'il agisse sur le plan privé ou sur le plan public, l'homme est libre de choisir ses modes de vie, mais il est responsable de l'ordonnance de son action, à la fois à sa propre vocation et au bien de la société tout entière ${ }^{12}$.

L'antimodernisme de Minville est par le fait même, sur le plan philosophique, un antilibéralisme. Il faut garder cette perspective en tête, car elle est d'une importance capitale pour comprendre la philosophie politique du Rapport Tremblay.

10. R. Arès, «Le commissaire royal», loc. cit., 694. Cela est confirmé par le procès-verbal de la réunion de la Commission Tremblay du 9 décembre 1954, Archives de l'École des Hautes Études commerciales de Montréal, fonds Esdras Minville, P035Z990004, dossier "Commission Tremblay 3 ".

11. Alexis Nouss, La modernité (Paris, Presses universitaires de France, coll. "Que sais-je?», $\left.\mathrm{n}^{\circ} 2923,1995\right), 29,31,55$.

12. Rapport Tremblay, op. cit., $2: 34$. 
Reste que ces normes intemporelles que nous venons d'évoquer, censées guider les hommes comme une étoile, ne se présentent jamais à eux dans toute leur pureté mais leur parviennent incarnées sous différentes formes. La nation, la culture, la tradition en constituent l'expression concrète, plus ou moins proche de l'idéal, en même temps que les courroies de transmission. Qu'est-ce qu'une nation pour Minville? C'est « une réalité sociologique, une communauté de culture - qui se forme et se renouvelle le long des années de la pratique en commun d'une même conception générale de la vie ${ }^{13}$ ». Une communauté humaine, donc, partageant une culture donnée, la culture nationale. Celle-ci est interprétation d'une philosophie universelle, c'est-à-dire une recherche «du bien, de la vérité, de la beauté, au service de l'homme en quête de réponse à ses plus hautes aspirations ${ }^{14}$ », particularisée par un milieu donné : histoire, géographie, "génie national», et transmise par un "milieu ethnique ${ }^{15}$ " (complexe d'institutions et de traditions nationales) où elle est d'usage courant. Elle constitue donc une certaine manière d'être humain, et, "sainement inspirée», est en ce sens un humanisme qui aspire à l'universel: «Toute culture nationale part donc de l'homme et du particulier pour déboucher dans l'humain et l'universel, conduire à l'un et à l'autre comme à un achèvement ${ }^{16}$.»

Qu'en est-il de la culture canadienne-française? C'est sans doute ici que l'influence de Lionel Groulx est la plus palpable. Les Canadiens français sont héritiers de la vieille culture française prérévolutionnaire que les colons de la Nouvelle-France ont emmenée dans leurs bagages à titre de culture vécue sous la forme de traditions, qui comme toutes les cultures vécues possèdent en elles le germe qui a le potentiel de faire naître de grandes œuvres. Inspiration chrétienne et génie français, épris d'ordre et de logique nous dit Minville, constitueraient la tonique et la dominante de cette culture ${ }^{17}$. Cette inspiration générale aurait donné naissance à une culture "qualitative», "spiritualiste», "personnaliste» et "communautaire ${ }^{18}$ ", traits qui sont l'essence de la culture canadienne-française - en même temps qu'un idéal à poursuivre. Mais la culture canadiennefrançaise n'est pas qu'une essence abstraite, elle est aussi particularisée

13. Ibid., 14.

14. Ibid., 8.

15. L'expression est du père Joseph-Thomas Delos, o.p.

16. Rapport Tremblay, op. cit., $2: 19$.

17. Ibid., 31.

18. Ibid., 32 . 
par un milieu, la vallée du Saint-Laurent principalement, et une histoire spécifiques, la conquête et ses suites ainsi que la dynamique économique nord-américaine, qui la différencient des autres ${ }^{19}$. C'est en quelque sorte la composante proprement américaine de la culture canadienne-française, qui n'affecte pas son essence mais en détermine la forme, pour reprendre le vocabulaire thomiste que Minville emploie ${ }^{20}$.

Minville conclut son étude en situant le problème des cultures au Canada dans le contexte plus large d'une «crise de civilisation» à laquelle ferait face l'Occident chrétien. Une civilisation humaniste et chrétienne pourra-t-elle survivre face à la montée d'une conception matérialiste et techno-scientifique de la vie, dans la foulée de l'extraordinaire développement économique et scientifique des dernières décennies? L'Occident chrétien saura-t-il dompter et assimiler ces forces économiques afin de se renouveler dans la continuité de sa tradition? Toute la difficulté consiste à combiner l'essentiel de la culture traditionnelle avec une judicieuse sélection de ce qu'offrent la science et la technique modernes: "Toute tentative de restauration sociale désireuse de demeurer dans la ligne d'inspiration de la culture canadienne-française doit partir de là [adaptation des traditions], non pour maintenir des modalités périmées, mais [...] pour créer selon l'esprit ancien celles qu'exigent les temps nouveaux ${ }^{21}$.»

Il faut choisir son camp: "Le cas canadien-français [...] n'est qu'un modeste épisode du vaste drame dans lequel sont engagés tous les peuples de même filiation culturelle et vraiment préoccupés de l'avenir de l'homme. Le monde de demain sera matérialiste [...] ou humaniste et chrétien, selon que les hommes d'aujourd'hui en auront décidé dans l'intimité de leur conscience $^{22}$. Canadiens français et Canadiens anglais sont du même camp dans cette crise, car ils ont en partage une même conception chrétienne de la vie - personnaliste, qualitative et spiritualiste. Ils doivent «chercher en commun ce qui, les unissant dans l'essentiel, peut leur permettre de se réaliser le plus complètement comme groupe ${ }^{23}$ ") dans le respect de leurs particularismes respectifs. Ainsi, l'existence du Canada français et la possibilité pour lui de se développer à sa manière conformément à son inspiration chrétienne et française rejoignent, à la racine, les questions les plus fondamentales de l'homme et de la civilisation, si modeste soit-il à leur

\footnotetext{
19. Ibid., 45-60.

20. Ibid., 15.

21. Ibid., 69.

22. Ibid., 86.

23. Ibid., 85 .
} 
échelle. Tout l'argumentaire développé dans le rapport pour justifier l'autonomie du Québec, jusqu'à la moindre réforme proposée, est tributaire de cette option fondamentale en faveur d'une culture spiritualiste et personnaliste dans le cadre de cette crise de l'Occident chrétien. Il apparaît clairement que refuser cette option et s'abandonner à une conception matérialiste du monde (un "effroyable cauchemar matérialiste» que Minville se refuse à envisager ${ }^{24}$ ) revient à miner l'édifice dans ses fondations les plus profondes. On comprend mieux, après ces éclaircissements, à quoi tient l'ambiguiité de la réception du Rapport Tremblay depuis la Révolution tranquille. D’une certaine manière, ceux qui se sont réclamés du rapport dans leurs luttes constitutionnelles et même dans leurs projets de réforme de toute sorte tout en rejetant ses fondements philosophiques n'ont pu que le dénaturer.

\section{2 - L'ART DE GOUVERNER: UNE CONCEPTION «CLASSIQUE-CHRÉTIENNE » DE LA POLITIQUE}

L'historien J. G. A. Pocock parlait jadis de l'importance d'interpréter les idées en les replaçant dans la bonne tradition intellectuelle afin d'en bien comprendre le contenu ${ }^{25}$. Cela nous paraît en effet être une notion méthodologique élémentaire en histoire des idées, que nous tâcherons d'appliquer sommairement dans cette section.

Quelle est donc la tradition de pensée politique à laquelle se rattache la Commission Tremblay? Il s'agit de la tradition que le politologue Philippe Bénéton nomme "classique-chrétienne ${ }^{26}$ » telle qu'elle est reprise et diffusée par l'Église, explicitée, notamment, par les grandes encycliques politiques du pape Léon XIII (1878-1903), puis par celles de Pie XII (19391958), et dont l'élément central est la synthèse aristotélico-thomiste ${ }^{27}$. Dans les grandes lignes, cette tradition politique est finaliste, idéaliste, moraliste, personnaliste, "prudente» et subsidiaire. Ces différentes notions s'éclaireront progressivement au fil de cette section.

\section{a) Les fondations: loi naturelle et personnalisme}

Pour bien comprendre la pensée politique du Rapport Tremblay, il faut remonter à ses fondements les plus profonds, à la conception de l'homme

24. Ibid., 83.

25. J. G. A. Pocock, "The History of Political Thought: A Methodological Enquiry", dans Peter Laslett et W. G. Runciman, dir., Philosophy, Politics, and Society (Second Series) (Oxford, Basil Blackwell, 1967), 200.

26. Philippe Bénéton, Introduction à la politique (Paris, Presses universitaires de France, 1997), 47.

27. D’où le qualificatif de pensée «classique-chrétienne». 
et du monde sur laquelle elle est construite. Dieu est l'auteur de l'univers. Dans cet ordre du monde, tout être, toute chose a une fonction bien précise à remplir et est investi des moyens d'accomplir cette tâche - sa fin. L'animal, par exemple, a pour objectif suprême de perpétuer l'espèce; il a l'instinct pour guide. Cette fin des êtres et des choses ainsi que les moyens de la réaliser constituent leur nature. Parce qu'ils sont le fruit de la volonté de Dieu, la nature de tout ce qui existe et cet ordre "organique» du monde sont donc considérés comme étant des biens objectifs dont la réalisation est l'objet essentiel de l'existence.

Un certain nombre de règles régissant cet ordre sont accessibles à la raison humaine, lui sont manifestées empiriquement: elles constituent la loi naturelle, qu'il faut entendre au sens aristotélico-thomiste. Ces préceptes dont la portée est universelle, qui sont les normes intemporelles dont nous parlions dans la section précédente demeurent plutôt généraux (on doit faire le bien, on doit honorer ses parents, on ne doit pas se faire justice soi-même, l'autorité est une nécessité en société humaine) et exigent d'être précisés et adaptés aux diverses circonstances par des lois particulières: c'est l'objet de la loi positive ${ }^{28}$.

Au sein de la Création, l'homme est un cas à part, tant du point de vue des fins que de celui des moyens. Il a d'abord la particularité d'être destiné à une fin surnaturelle, son existence devant en définitive être consacrée à rendre son âme digne du Ciel après la mort physique. Il a ensuite le privilège de pouvoir choisir les moyens de réaliser cette fin ultime, comme ses fins temporelles intermédiaires. Autrement dit, Dieu a fait la nature humaine libre et raisonnable. Outre la Révélation, c'est la loi naturelle qui nous l'apprend: le fait de la raison humaine révèle en effet que Dieu a voulu que l'homme se conduise à sa fin librement, par ses propres moyens - sans quoi il ne l'eût doté que d'un fort instinct comme dans le reste du règne animal. L'homme, écrit Minville, "doué d'intelligence et de volonté libre», est "appelé à une vocation personnelle dont il est l'artisan et le seul responsable devant le temps et devant l'éternitée ${ }^{29}$ ". Il doit se guider d'après la raison et s'adapter aux diverses circonstances dans lesquelles il est plongé. Sa liberté est ordonnée à plus grand que lui : «Vivre librement, ce n'est pas vivre à sa guise, mais vivre selon les exigences de

28. Marie-Odile Métral-Stiker, "Naturel (Droit)», Encyclopédie Universalis, 2005, www.universalis.edu, consultée le 26 octobre 2005 ; Richard Arès, Petit guide moral du législateur (Montréal, École sociale populaire, brochure $\left.\mathrm{n}^{\circ} 350,1943\right), 13-19$.

29. Rapport Tremblay, op. cit., $2: 32$. 
l'ordre tel qu'il a été établi par la loi naturelle, elle-même illuminée par la Révélation ${ }^{30}$ ", écrit encore Minville.

Tout ne se vaut pas dans la vie de l'homme: les valeurs de l'esprit et sa vocation surnaturelle ont la primauté sur les fins utilitaires ${ }^{31}$. Le bien humain est avant tout «spiritualiste» et antimatérialiste:

l'homme est avant tout un être spirituel, intelligent et libre, doué d'une âme immortelle, bref une personne qui, comme telle, dépasse en valeur tout l'univers matériel et a la charge et la responsabilité de sa propre destinée. [...] Une société à caractère vraiment politique cherchera à respecter et à promouvoir cette vie de l'esprit chez ses membres ${ }^{32} \ldots$

Dans l'esprit des commissaires, prendre en charge son propre destin temporel et spirituel, c'est-à-dire s'acquitter de sa tâche consciencieusement, avec intégrité et dévouement, créer un milieu familial favorable au développement de ses enfants, être prévoyant, gagner son ciel en honorant Dieu, mettre en valeur toutes ses facultés, soit les dons que Dieu a confiés à chacun, agir par soi-même, valent en eux-mêmes et constituent une condition irremplaçable au plein accomplissement de la personne.

\section{b) Une politique finaliste}

À partir de ces prémices s'élabore une pensée politique dite finaliste, c'està-dire que la politique est dotée d'une fin propre, fondée en nature donc indépendante de la volonté humaine, et est intégrée à cet ordre du monde créé par Dieu qui dépasse l'homme ${ }^{33}$. Dans cette perspective finaliste, tout ce qui concerne la politique doit être ordonné à sa fin, comme c'est d'ailleurs le cas pour tout ce qui existe. Comme cette fin ne peut être altérée par l'homme, la volonté de l'État ne saurait constituer à elle seule le droit, non plus que la volonté du peuple (ou de qui que ce soit), d'où l'opposition irréductible des catholiques à la thèse de la souveraineté du peuple ${ }^{34}$ et à ce qu'on appelle le positivisme juridique. Lisons Charles de Koninck, qui traduit en termes politiques le passage de Minville cité dans la sous-section précédente:

30. Ibid., 34 .

31. Ibid., 32 et 34 .

32. Ibid., 111 et 276 .

33. P. Bénéton, Introduction à la politique, op. cit., 61.

34. Cette opposition n'est aucunement incompatible avec la participation du peuple au gouvernement de la cité, comme en témoigne l'œuvre de saint Thomas d'Aquin lui-même. Le véritable problème est bien plutôt qu'un pouvoir humain déclaré «souverain " ne soit redevable de ses actes devant personne - et surtout ni devant Dieu, donc ait le champ libre pour soumettre la société à sa tyrannie, craint-on. 
Pour être admissible, la souveraineté, dans l'ordre politique, doit être relative. Si la vie en communauté politique a pour but la poursuite du bien humain parfait, c'est dans cet ordre restreint que l'autorité doit être souveraine; souveraine non pas quant à la détermination de ce qui constitue le bonheur de l'homme ni par rapport à la loi ni au droit naturels, mais souveraine quant aux moyens pratiques, très concrets, de rendre ce bien accessible ${ }^{35}$.

Par l'ampleur de son objet, de la fin qu'il poursuit, la politique trône au sommet de la vie temporelle - sans que celle-ci ne s'y résume. Quel est cet objet? Le bien commun, fin suprême de l'ordre temporel. En quoi consiste ce bien commun dont la société politique a la responsabilité? Minville le définit comme "l'ensemble organisé des biens nécessaires à l'accomplissement de la vocation de l'homme - sur le plan politique ${ }^{36}$ ». Dans le même sens, on lit ce qui suit en conclusion: "Dans son acception la plus haute, la politique a pour objet non seulement le bien-être mais le bien-vivre, c'est-à-dire l'ensemble hiérarchique des conditions nécessaires à la pleine affirmation de la personne humaine. Si le citoyen est au service de l’État, celui-ci est au service de l'homme ${ }^{37}$." L'épanouissement de l'homme, de la personne humaine conçue dans la perspective chrétienne, est en somme la fin ultime de la politique, par le truchement du bien commun: «Bref, l’homme lui-même considéré dans l'intégrité de son être et de sa fin est la donnée ordonnatrice de l'ordre social ${ }^{38}$. »

“Euvre de pensée, œuvre d'hommes conscients ${ }^{39}$ ", la politique est l'aboutissement et la plus haute expression de la capacité de l'homme à se gouverner par la raison. Grâce à elle, ce dernier peut harnacher le puissant mouvement de la vie économique et sociale dans le sens du bien commun: "il appartient à la politique de diriger l'activité économique, dont l'objet est le bien-être, et de créer les conditions de progrès qui le maintiennent en accord avec les exigences du bien-vivre - objet premier de la vie en sociétét0".

35. Charles de Koninck, La Confédération, rempart contre le Grand État, annexe 1 du Rapport Tremblay, 11. Il est bien précisé, dans la présentation de chaque annexe du rapport, que les opinions qui y sont défendues n'engagent que leurs auteurs et non la Commission. Cependant, il nous paraît évident que les thèses de Charles de Koninck sont tout à fait conformes aux bases philosophiques du Rapport Tremblay. Pour cette raison, nous nous permettons de considérer cette annexe comme partie intégrante du rapport sur le plan de la pensée politique et la citons comme telle. Nous traitons de même les annexes de François-Albert Angers.

36. Rapport Tremblay, op. cit., $2: 14$.

37. Ibid., 3-II : 299. Nos italiques.

38. Ibid., 3-I: 116.

39. Ibid., 3-II: 324 .

40. Ibid., $2: 86$. 
Tout, dans l'ordre politique comme dans l'ordre économique ou social, doit donc être mesuré à l'aune de cette conception spiritualiste de la personne humaine et ordonné à la réalisation de sa vocation naturelle et, au-delà, surnaturelle. Il s'agit là, pour les commissaires, de la finalité ultime de tout l'ordre temporel. Cette fin personnaliste de l'ordre temporel est d'ailleurs une des plus importantes innovations du christianisme et un des héritages les plus précieux et durables qu’il ait légués ${ }^{41}$.

\section{c) État, communautés humaines et subsidiarité}

L'homme, d'après cette conception, est social par nature ${ }^{42}$. Il s'insère dans une myriade de communautés (famille, profession, associations diverses, Église) et s'unit à ses semblables pour former société car c'est là la seule manière pour lui de se réaliser complètement - un être isolé étant voué à une existence fortement diminuée et limitée. Ces communautés, nous dit le père Arès, produisent toutes leur bien propre et contribuent à leur manière à la réalisation du bien commun, quoique aucune "n'épuise les exigences et les possibilités sociales de [la] nature [de l'homme]», pas même l'État. La nation, considérée comme une "communauté de culture» dans le Rapport Tremblay, nous l'avons vu, est un de ces groupements les plus importants. Elle fournit à l'homme "un style de vie, une manière de se réaliser lui-même, de parvenir à la plénitude de son être ${ }^{43}$ ", et à ce titre est responsable d'un bien humain de premier ordre qui est partie intégrante du bien commun. Ainsi, l'État, responsable suprême du bien commun, «doit favoriser la conservation et l'épanouissement des valeurs de culture dont la nation apporte à l'homme le bienfait ${ }^{44}$ ". C'est par cette logique que la nation, fait en soi culturel, devient objet de la politique. Minville décrit ce phénomène de manière limpide:

41. P. Bénéton, Introduction à la politique, op. cit., 39; Marcel Prélot, Histoire des idées politiques (Paris, Dalloz, 1970), 135. Le passage suivant de saint Thomas d'Aquin, tiré de l'opuscule De regno, témoigne de l'influence de la pensée du Docteur angélique sur les auteurs du Rapport Tremblay: «En effet, les hommes se réunissent pour mener en commun une vie bonne, but que ne peut atteindre l'homme isolé. Or la vie bonne c'est la vie selon la vertu. [...] Mais, puisque l'homme, en vivant selon la vertu, est ordonné à une fin ultérieure qui consiste [...] en la jouissance de Dieu, il faut que la société humaine ait une fin identique à la fin personnelle de l'homme. La fin dernière de la société n'est donc pas la vie vertueuse mais, par cette vie vertueuse, de parvenir à la jouissance de Dieu. » Saint Thomas d'Aquin, Du gouvernement royal [De regno], I: 14. (Traduction: Claude Roguet).

42. Selon l'esprit de la célèbre formule d'Aristote : l'homme est un animal civique.

43. Rapport Tremblay, op. cit., 2: 14.

44. Ibid., 22. 
Une nation n'est assurée de son destin que si elle est parvenue ainsi à informer de son esprit les grandes fonctions de la vie collective ressortissant à l'autorité politique et du même coup à établir l'accord entre ses effectifs humains et leur milieu social. C'est pourquoi sans être de sa nature ni une réalité économique, ni une réalité politique, elle est induite par les exigences mêmes de son maintien et de son progrès à s'engager dans l'action économique et l'action politique - à se poser ainsi en face de l'État et à exiger de lui certaines attitudes ${ }^{45}$.

Sont ainsi posées les balises d'un nationalisme politique légitime, où le bien national est pris en considération par l'autorité politique mais n'est jamais substitué au bien commun de la population en général comme fin suprême d'une société. C'est pour cette raison qu'un Richard Arès par exemple, pourtant profondément nationaliste, se garde de réduire l'État québécois au seul statut d'État national des Canadiens français, suivant en cela l'esprit général de la doctrine catholique. Il s'agit assurément d'un équilibre subtil difficile à réaliser concrètement. L'organisation fédéraliste doit aider à y parvenir. Notons toutefois que cette question des relations entre État et nation pose problème tout au long du rapport. Tentant de trouver le juste milieu entre primauté du bien commun et nécessité de tenir compte du bien national comme composante du bien commun, les commissaires naviguent d'une conception neutre du rôle de l'État en ce domaine à une conception selon laquelle l’État est "nationalisé», voire monopolisé de façon naturelle par la nation majoritaire. Il y a là, non pas contradiction, mais une certaine difficulté dans l'idéologie, qui n'est pas neuve d'ailleurs (déjà la thèse de l' «État français» de Groulx présentait cette ambiguïté) et qui provoquera certaines tensions entre nationalistes traditionalistes durant la Révolution tranquille, comme le montre la thèse de Xavier Gélinas ${ }^{46}$.

«[L]e fait naturel et multiforme de la sociabilité humaine, de la variété et de la complexité de la vie sociale » reflète la diversité des hommes euxmêmes et est le fruit naturel de l'expression de leur liberté ainsi que des besoins humains. Fait de nature et de liberté, cette variété est donc bonne en soi et doit être encouragée, car si l'homme ne peut se réaliser qu'avec l'appoint de la société, il est aussi et avant tout lui-même responsable de son plein épanouissement, et seule une société qui se développe conformément à cette donnée personnaliste essentielle est réellement en

45. Ibid., 21.

46. X. Gélinas, La droite intellectuelle québécoise..., op. cit., 364-462. 
mesure d'atteindre sa finalité et de produire le véritable bien de la personne $^{47}$.

Cette conception de l'homme et de la société est au fondement du principe central de subsidiarité, pierre de touche de la doctrine sociale et politique de l'Église. Le pape Pie XI (1922-1939) en a donné une définition, devenue classique, dans l'encyclique Quadragesimo anno (1931), reprise depuis par tous les catholiques sociaux. Il n'est pas inutile de la rappeler ici:

de même qu'on ne peut enlever aux particuliers, pour les transférer à la communauté, les attributions dont ils sont capables de s'acquitter de leur seule initiative et par leurs propres moyens, ainsi ce serait commettre une injustice, en même temps que troubler d'une manière très dommageable l'ordre social, que de retirer aux groupements d'ordre inférieur, pour les confier à une collectivité plus vaste et d'un rang plus élevé, les fonctions qu'ils sont en mesure de remplir eux-mêmes ${ }^{48}$.

Le Rapport Tremblay se montre digne héritier de cette doctrine: «à l'égard des individus et des groupements inférieurs, toute collectivité doit se contenter d'exercer une fonction supplétive et subsidiaire, se gardant en conséquence de faire à leur place ce qu'il sont capables de faire par eux-mêmes ${ }^{49}$ ». Une société différemment conçue, dans laquelle les groupements supérieurs assumeraient les responsabilités qui devraient normalement revenir aux groupements inférieurs ou à l'individu, serait en quelque sorte «contre-nature » et constituerait assurément un amoindrissement pour la personne humaine, ainsi privée d'une partie de son agir. L'État demeure l'organe suprême de la société, sur le plan temporel, mais ses fonctions relèvent ainsi davantage du contrôle et de la coordination des actions de la société civile:

L’État, dans ses fins ultimes, s'adresse à l'homme en tant qu'homme, le "gouverne", c'est-à-dire l'encadre, le soutient, le supplée au besoin, disposant toute chose de telle façon qu'il puisse, de son initiative propre ou par l'intermédiaire des communautés qui naissent de la pratique quotidienne de la vie, s'assurer les biens nécessaires à la réalisation de sa $\mathrm{fin}^{50}$.

47. Rapport Tremblay., op. cit., 2 : 112-115.

48. Pie XI, Quadragesimo anno, dans Émile Marmy, dir., La communauté humaine selon l'esprit chrétien (Fribourg/Paris, Éditions St-Paul, 1949 [1931]), 386. Pour une bonne étude sur le sujet, voir Chantal Millon-Delsol, Le principe de subsidiarité (Paris, Presses universitaires de France, coll. "Que sais-je?», 1993), 127 p.

49. Rapport Tremblay, op. cit., $2: 116$.

50. Ibid., 14. 
Cette conception n'est donc pas à proprement parler radicalement antiétatiste, puisqu'elle laisse ouverte la possibilité d' "interventions résolues et hardies de l'État au nom du bien commun ${ }^{51}$ ", comme en témoigne l'exhaustivité des recommandations du rapport. Cependant, elle tient pour une règle d'or que toute intervention de l'État doit laisser autant de place que possible à l'initiative personnelle, ait constamment le souci de l'encourager et se retire du jeu dès que celle-ci est en mesure d'assumer seule ses responsabilités ${ }^{52}$. Il s'agit là en fait d'un étatisme souple et très modéré donnant la priorité à l'initiative personnelle, qui aspire à trouver un juste milieu entre l'antiétatisme poussé voire radical du libéralisme classique et de l'anarchisme d'un côté, et l'interventionnisme à grande échelle du socialisme et de l'État-providence de l'autre. Le principe de subsidiarité, au fond, aspire à donner corps à la société et à l'exigence du bien commun qui est sa fin tout en respectant la nature libre et responsable de l'homme ainsi que la diversité naturelle de la vie sociale. Dans l'esprit des commissaires, c'est, pour un chrétien, la seule conception de la société envisageable.

\section{d) Prudence et art politique}

Cette irréductible complexité des sociétés humaines, couplée aux limites de la raison ainsi qu'à la conception finaliste de la politique qui aspire à un bien normatif, non pas seulement à l'efficacité, fait que la politique telle que conçue dans le Rapport Tremblay ne se réduit pas à une technique, dans une perspective rationaliste, mais consiste plutôt en un art, suivant en cela l'esprit général de la tradition politique classique-chrétienne et de l'aristotélico-thomisme. Philippe Bénéton précise ainsi ce qu'il faut entendre par «art» politique : «une activité pratique $[\ldots]$ ordonnée à des fins naturelles qui la définissent et lui donnent son sens, [qui] requiert pour accomplir ces fins un savoir sans doute technique mais aussi personnel (le "tour de main", le discernement né de l'expérience) ${ }^{53}$ ". C'est que la réalité est si complexe et mouvante que la raison humaine, aussi puissante soit-elle, est incapable de la saisir intégralement dans toutes ses ramifications; son infinie diversité est irréductible à tout système conçu par la raison, qui jamais ne l'épuisera. Or la politique consiste précisément à conduire des hommes concrets au travers des conditions les plus diverses vers un bien objectif, qui fixe les paramètres généraux de cette démarche.

51. Ibid., 315.
52. Ibid., 316.
53. P. Bénéton, Introduction à la politique, op. cit., 13 . 
Tout l'art du politique est de savoir conjuguer ces grands principes généraux déduits de la finalité à atteindre aux conditions concrètes les plus diverses, de créer la meilleure synthèse de ces principes et de la réalité, et de savoir la faire évoluer au même rythme que cette réalité pour éviter l'obsolescence et pour que l'organisation de la société mette toujours la personne en état d'accomplir sa fin.

Cette aptitude particulière, "vertu cardinale» du bon politique, a pour nom prudence dans la synthèse aristotélico-thomiste, "prudence» qui ne s'entend pas au sens courant du terme mais se définit plutôt comme sagesse pratique, raison pratique, «discernement pratique ${ }^{54}$ » caractérisant en politique une capacité particulière à saisir les grands courants changeants de la vie temporelle et à les orienter vers le bien commun et le bien de la personne. Il y a une bonne part d'instinct, de sens commun et d'empirisme dans cette vertu, qui en font bien davantage qu'une pure capacité de raisonnement, sans que celle-ci en soit pour autant exclue. Dans le même registre, il faut noter l'importance que revêt la moralité personnelle des dirigeants, leurs propres qualités, dans cette conception de la politique. Comme l'écrit Charles de Koninck:

En dernière instance, la vie politique ne peut être sauvée que par la qualité de l'individu - par sa tempérance, sa force, sa justice, et sa prudence personnelle, domestique, politique. Vouloir compter sur un "système" pour contourner la difficulté du bien-agir, c'est le faux espoir du défaitisme - l'attitude typiquement «réactionnaire» de l'avant-garde socialisante ${ }^{55}$.

C'est là un pli typique de la pensée politique d'un catholique: ce qui compte avant tout dans ce domaine est la qualité personnelle des gouvernants ainsi que l'inspiration générale du régime, c'est-à-dire sa conformité ou non à la conception chrétienne de l'homme et de la société. Les structures, quant à elles, sont perçues comme étant d'une importance secondaire, au point où plusieurs en viennent à les négliger purement et simplement. Poussée trop loin, cette logique peut avoir l'effet paradoxal de produire une pensée politique désincarnée alors que c'est précisément ce que la notion de prudence politique cherche à éviter. Les intellectuels canadiens-français, en général, sont loin d’avoir été imperméables à ce péril... Dans le Rapport Tremblay toutefois, les importants développements consacrés au fédéralisme sauvent la mise.

54. Ibid., 51.

55. C. de Koninck, La Confédération..., op. cit., 35. 
On a pu trouver un peu étrange cette description de la conception classique-chrétienne de la politique jusqu'à maintenant, qui parle de l'inaliénable liberté de l'homme d'un côté tout en affirmant de l'autre l'existence objective de normes intemporelles et d'un ordre du monde qui s'impose à l'homme et dont les fins sont soustraites à sa volonté. C'est que nous étions dans l'ordre des fins, justement, qui échappent au contrôle de l'homme. La liberté de celui-ci est bien réelle, mais elle ne s'exerce qu'au niveau des moyens à mettre en œuvre pour réaliser cette fin. En politique, c'est la vertu de prudence qui fixe les paramètres de l'exercice de la liberté humaine d'après la conception chrétienne: les fins et les principes généraux qui en découlent sont intemporels, mais il appartient à l'homme de décider des meilleurs moyens pour les mettre en œuvre hic et nunc, ici et maintenant. C'est à ce niveau que la liberté humaine joue. La question du régime politique - donc celle des structures - est l'objet du plus important de ces choix politiques dont l'homme a la responsabilité. Ce choix est œuvre par excellence de prudence, nous dit Charles de Koninck, qui «doit tenir compte de la nature et de l'histoire de la patrie, du caractère donné du citoyen ${ }^{56}$ », donc de l'ensemble des caractères d'une société donnée. D’une manière générale, c'est toute la politique qui doit être menée dans cet esprit de prudence - politique sociale, économique, etc. La notion de subsidiarité y est tout à fait conforme en ceci qu'elle ne fixe pas à l'État de règle fixée dans le béton, de formule rigide qui lui prescrirait dans toute situation l'action concrète à entreprendre: l'État intervient ou non au gré des circonstances, selon ce qu'exige le bien commun $^{57}$. La souplesse de cette approche, qui est sa force, fait cependant aussi sa faiblesse en ceci que toutes sortes de positions, des plus interventionnistes à celles qui le sont le moins, peuvent se réclamer de la subsidiarité. Il n'est en effet pas facile de déterminer concrètement le moment où l'initiative individuelle devient insuffisante et doit être suppléée par l'intervention de l’État...

\section{3 - UNE PENSÉE FÉdÉRALISTE}

La pensée fédéraliste du Rapport Tremblay a un visage et porte un nom, ceux d'un docte et cérébral jésuite devenu à force de travail un pionnier

56. Ibid., 4.

57. Rapport Tremblay, op. cit., 3-I : 118. Dans le même sens, Marc Chevrier écrit: «À vrai dire, de tous les concepts organisateurs que nous livre la théorie politique, [le principe de subsidiarité] est peut-être celui qui nous demande de penser le rôle de l'État avec le moins d'a priori." M. Chevrier, «La conception pluraliste... », loc. cit., 54. 
de la science politique dans notre milieu : le père Richard Arès, s.j. C'est dans le fameux deuxième volume du rapport que celui-ci expose sa pensée sur ce qui constitue l'un de ses sujets de prédilection ${ }^{58}$.

Précisons, avant d'aborder le sujet de front, que la dichotomie fédéralisme/souverainisme qui caractérise le débat constitutionnel dans le Québec d'aujourd'hui ne peut rendre compte adéquatement des débats des années 1950. Au contraire, elle brouille les cartes en tendant à associer sans nuance fédéralisme et centralisme. En fait, les nationalistes canadiensfrançais des années 1950, autonomistes québécois convaincus, se considèrent les véritables défenseurs du fédéralisme au Canada - cela est très clair à la lecture du Rapport Tremblay. Pour caractériser le débat constitutionnel dans le Québec de l'époque, il serait plus approprié de parler d'opposition centralisme/autonomisme (ou provincialisme) ${ }^{59}$.

Pour le père Arès, le fédéralisme au sens le plus général est le «régime de l'association " qui a pour principale caractéristique et valeur de trouver un juste milieu entre l'exigence de l'unité et le respect des individualités ${ }^{60}$. On reconnaît en cela le souci, fondamental pour la pensée politique d'inspiration chrétienne, de concilier la nécessité d'une direction de la société vers le bien commun et l'autonomie des groupes et des personnes. Dans son étude publiée dans le Rapport Tremblay, le jésuite traite tout d'abord du fédéralisme comme système politique et juridique (son sens premier), puis élargit le questionnement et aborde le fédéralisme comme «fait social».

\section{a) Fédéralisme politique et juridique}

Le fédéralisme comme système politique et juridique est par excellence le régime du compromis entre forces centripètes et centrifuges, permettant «l'union dans la diversité». Arès en propose la définition suivante: «régime d'association entre États dans lequel l'exercice de la puissance étatique se partage entre deux ordres de gouvernement, coordonnés mais non subordonnés entre eux, chacun jouissant du pouvoir suprême dans

58. Voir notamment la série d'articles qu'il a publiés dans la revue L'Action nationale: «Défense et illustration du fédéralisme», octobre 1950, 95-109; "Fédéralisme et organisation de la vie sociale», novembre 1950, 169-185; «Fédéralisme et organisation de la vie politique», décembre 1950, 253-269; «Fédéralisme et centralisation », janvier 1951, 5-14.

59. En réalité, cette division marque encore de nos jours le camp fédéraliste. Simplement, la question de l'indépendance du Québec a recentré le débat autour d'un affrontement entre un camp "Québec» et un camp «Canada», gommant par le fait même ces divergences, potentiellement très importantes.

60. Rapport Tremblay, op. cit., 2: 109. 
la sphère d'activité que lui assigne la constitution ${ }^{61}{ }$. L'État fédéral est donc composé d'un gouvernement central et de gouvernements régionaux qui sont chacun maîtres dans l'exercice des pouvoirs que la constitution fédérale leur assigne. Pour que ce partage ne soit pas une fiction, chacun des deux ordres de gouvernement doit être indépendant de l'autre, notamment du point de vue fiscal. Arès rejoint ainsi le propos général du rapport. Puis, il traite des principales caractéristiques de l'État fédéral, soit le partage des pouvoirs et leur stricte délimitation, la suprématie de la constitution et, en conséquence, celle des tribunaux.

Cependant, l'idée la plus importante qui sous-tend tout ce développement sur le fédéralisme politique est l'opposition à une interprétation stricte du principe des nationalités, selon lequel toute nation (au sens culturel du terme) doit, pour se réaliser pleinement, être dotée d'un État entièrement indépendant, comme si l'aspiration au rang d'État-nation devait être une nécessité de principe. À vrai dire, il ne fait qu'évoquer cette idée dans le rapport, mais elle n'en demeure pas moins très présente, par exemple lorsqu'il parle de l'importance du compromis entre tendance unitariste et tendance séparatiste au sein d'un État. D’ailleurs, il consacre de fortes pages de son ouvrage Notre question nationale à cette question du principe des nationalités ${ }^{62}$. Celui-ci, dans son esprit, ne peut mener qu'à une impasse s'il est pris au sens absolu en dressant l'une contre l'autre dans un affrontement sans merci deux tendances contraires: l'État central travaillant à laminer toutes les différences au profit d'une nationalité unique d'un côté, les nationalités cherchant à gagner leur indépendance politique à tout prix, faisant ainsi peser la menace du morcellement infini sur les États et de l'anarchie dans la vie internationale, de l'autre. Il y a en outre dans ce principe une confusion malheureuse entre le bien commun et le bien national, qu'un tenant de la doctrine catholique se doit d'éviter. Pour le père Arès, il est clair que seul le fédéralisme permet de sortir de cette impasse en conciliant le besoin d'unité des uns et l'aspiration à l'autonomie des autres, tout en maintenant la distinction et la hiérarchie entre bien commun et bien national. Par ailleurs, il reconnaît la validité du principe des nationalités entendu dans un sens modéré et non dogmatique, c'està-dire qu'il est à ses yeux souhaitable que les nations jouissent d'une certaine mesure d'autonomie politique au sein d'un État plurinational, ou à

61. Ibid., 98.

62. Richard Arès, Notre question nationale, 2 : Positions de principe (Montréal, Éditions de l'Action nationale, 1946), 74-115. 
tout le moins d'une reconnaissance et d'une protection juridiques, dans le cas où elles ne pourraient aspirer à l'indépendance complète sans que cela ne cause plus de problèmes qu'il n'en règle. Il paraît faire sien le nationalisme politique modéré tel que Minville le définit dans une autre partie du rapport et que nous avons présenté dans la deuxième section ${ }^{63}$.

\section{b) Fédéralisme social}

Mais le fédéralisme, dans l'esprit du père Arès, est bien plus qu'un simple système d'organisation politique et juridique: il se présente également comme un "système général d’organisation de la société64 ${ }^{4}$. Cette section sur le «fédéralisme social ${ }^{65}$ » est le cour de l'étude du père Arès. Le fédéralisme, avant de s'incarner dans des structures politiques, est un esprit qui informe la culture et l'organisation de toute la société :

Le fédéralisme, comme régime politique et juridique, ne pourra se maintenir et donner satisfaction qu'à la condition de pousser ses racines jusque dans la vie sociale elle-même et d'y rencontrer des bases sociologiques et même philosophiques sur lesquelles il pourra fermement s'appuyer. En d'autres termes, le véritable esprit du fédéralisme, c'est par delà les structures politiques et juridiques qu'il faut le chercher ${ }^{66}$.

Pour Arès, la principale valeur de l'ordre fédéraliste, du fédéralisme comme organisation sociale, tient à ce qu'il « cherche à traduire pratiquement la conception chrétienne de l'homme tout en tenant compte de la variété et de la complexité de la vie sociale ${ }^{67}$ ». Cet ordre fédéraliste est appuyé sur quatre piliers philosophiques et sociologiques. D’abord, sur

63. Notons que le père Arès évolue sur cette question au fil des ans et assouplit sa position durant les années 1960 en admettant la thèse du statut particulier pour le Québec au sein de la fédération canadienne. Cette évolution est évoquée par Pierre Trépanier, «Richard Arès», L'Action nationale, 82,2 (février 1992): 167-198, ainsi que par X. Gélinas, La droite intellectuelle québécoise..., op. cit. Il est vrai, cependant, qu'on trouve en germe l'idée de «statut particulier» dans le Rapport Tremblay. On y parle en effet de la «mission particulière» du Québec au sein du Canada. Rapport Tremblay, op. cit., 3-I: 4.

64. Rapport Tremblay, op. cit., 2: 107.

65. Dans un article publié en 1950, Arès écrit que cette notion élargie de fédéralisme est entre autres inspirée des travaux d'Alexandre Marc sur le «fédéralisme intégral» ainsi que sur l'œuvre de l'essayiste fédéraliste et conservateur suisse Gonzague de Reynold. Voir R. Arès, «Fédéralisme et organisation de la vie sociale», loc. cit., 170. Par ailleurs, il semble qu'il faille attribuer la paternité de cette idée de "fédéralisme social» à Pierre Joseph Proudhon. Voir Bernard Barthalay, Le fédéralisme (Paris, Presses universitaires de France, coll. "Que sais-je?», n 1953, 1981), 54-71. Arès ne cite pas directement Proudhon, mais fait mention d'une étude d'Yves Simon sur cet auteur publiée dans la revue Esprit. Rapport Tremblay, op. cit., 2: 117.

66. Rapport Tremblay, op. cit., $2: 107$.

67. Ibid., 118. 
la conception chrétienne de l'homme et des fins de la société. Selon cette conception, l'homme, nous l'avons dit, est un être libre, raisonnable et spirituel qui a la responsabilité première de la réalisation de ses fins naturelle et surnaturelle. L'objet de la société, nous dit Arès, ne serait donc pas tant de conduire l'homme que de lui apprendre à se conduire lui-même ${ }^{68}$. Deuxième principe fondateur: le fait de la diversité de la vie sociale, lui aussi exposé dans la troisième section de ce chapitre. Le fédéralisme "part de la vie sociale telle qu'elle est et il l'admet dans toute sa variété et sa complexité. Son but ce n'est pas d'asservir, mais de coordonner, ce n'est pas d'unifier, mais d'unir ${ }^{69}$ ». En fait, il prend appui sur cette diversité et respecte l'autonomie des personnes ainsi que des divers groupes qui composent la société. Troisième principe : une conception pluraliste du bien commun, «aussi varié et complexe» que la société l'est elle-même ${ }^{70}$. À une conception moniste du bien commun qui relèverait de la seule responsabilité de l'État, Arès oppose un bien commun conçu comme «un ensemble organisé de cercles concentriques qui entourent la personne humaine, allant de la famille à la société internationale, et se réalisant par degrés, par étapes et par paliers ${ }^{71}$ \%. Chacune des communautés composant la société, fruit de la sociabilité naturelle de l'homme que nous avons évoquée précédemment, poursuit son bien propre qui contribue au bien commun général. L'État n'a pas le monopole de celui-ci. Cette conception assure la préservation d'une sphère privée échappant à la volonté de l'État, condition essentielle à la liberté de l'homme. Le quatrième principe découle des précédents: il s'agit du principe de subsidiarité. Puisque l'homme a besoin du concours de la société pour atteindre sa fin, celle-ci doit l'y aider mais en respectant sa responsabilité première à cet égard. En conséquence les groupes constituant la société n'agiront que pour suppléer aux insuffisances des individus et des groupes inférieurs, jusqu'à l'État, organe suprême de la société sur le plan temporel.

Ces principes devront se traduire concrètement par une organisation décentralisée de la vie politique, par la reconnaissance tant juridique que pratique des autonomies régionales ainsi que par la collaboration des différentes nationalités dans le respect de leurs différences (ceci tendant à éclipser cela en importance, comme on peut s'en douter...). L'esprit de décentralisation dans le domaine politique se traduit par l'application du

\footnotetext{
68. Ibid., 111.

69. Ibid., 113.

70. Ibid., 113.

71. Ibid., 114.
} 
principe de subsidiarité et a pour but de mettre la personne en état d'exercer toutes les responsabilités qu'elle est capable d'assumer. C'est là la marque d'une organisation sociale qui vise avant tout à élever l'être humain et à le porter à ses sommets: "Ce que [le fédéralisme] veut assurer, c'est un organisme dont toutes les parties soient pleines de vie et se gèrent elles-mêmes, et non pas un mécanisme à ressort central omnipotent $^{72}$.» La reconnaissance juridique par l'État des différentes communautés formant la société fonde en droit l'autonomie des corps intermédiaires et protège efficacement leur liberté contre la volonté toute-puissante de l'État. C'est une manière particulière d'institutionnaliser la séparation des pouvoirs, au fond, et d'éviter qu'il soit trop concentré - entre les mains de l'État, ce qui est aux yeux du jésuite le péril de l'heure. Arès n'aborde pas dans son étude les problèmes liés à une organisation décentralisée de la société. Charles de Koninck le fait, cependant, dans son annexe : il parle de «l'inconvénient de l'injustice par faveur» (favoritisme lié au patronage) qui caractérise ce qu'il appelle le «Petit État», par opposition à «l'injustice par classification » qui caractérise le «Grand État » - c'est-à-dire la propension de ce dernier à tout uniformiser, à traiter de la même manière des réalités pourtant différentes, son incapacité à tenir compte de la diversité de la vie sociale ${ }^{73}$. Il est incontestable que le philosophe de l'Université Laval préfère, et de loin, courir le risque de la première injustice que celui de la seconde, ce qui est sans doute également l'option du père Arès.

Les lecteurs familiers avec les textes d'intellectuels canadiens-français des années 1930 à 1960 auront tout de suite saisi la référence implicite au corporatisme dans ces développements concernant la reconnaissance légale des corps intermédiaires. Depuis les années 1930 en effet, on fait la promotion dans les cercles traditionalistes canadiens-français de cette ambitieuse réforme consistant à décentraliser certains pouvoirs en matière économique et sociale vers des conseils professionnels regroupant sur une base paritaire patrons et ouvriers dans chaque grand secteur économique. C'est la voie que privilégient de nombreux catholiques sociaux pour assurer un certain contrôle de l'économie, tout en respectant le plus possible l'autonomie de la personne ainsi que le principe de subsidiarité. En fait, le mot «corporatisme» ainsi que ses dérivés et synonymes (comme "organisation professionnelle») sont à peu près absents du Rapport Tremblay, pour une raison qui parait évidente : les commissaires veulent

72. Ibid., 121.

73. C. de Koninck, La Confédération..., op. cit., 16. 
éviter de discréditer leur rapport en l'associant à un concept que les expériences fascistes ont rendu odieux aux yeux de plusieurs ${ }^{74}$. Marc Chevrier, dans l'article qu'il consacre à la Commission Tremblay, écrit qu'il est nécessaire de bien distinguer le principe de subsidiarité du corporatisme, rejetant celui-ci de manière catégorique sous prétexte qu'il tenterait de s'arroger le monopole de la puissance publique, contre l'exigence de pluralisme du principe de subsidiarité ${ }^{75}$. On ne tranchera pas ici le difficile débat de la vraie nature du corporatisme. Cependant, il faut bien réaliser que les trois figures intellectuelles principales de la Commission Tremblay, Minville, Arès et Angers, sont des corporatistes sociaux convaincus et le demeurent au moins jusqu'à l'aube des années 1960 - et sans doute audelà. Ils ne voient assurément aucune incompatibilité entre le corporatisme social, hérité de la tradition catholique sociale, et le principe de subsidiarité, bien au contraire, et n'ambitionnent certainement pas de faire des corporations les corps politiques hégémoniques de la société, comme en témoigne de façon patente l'étude d'Arès sur le fédéralisme. La profession organisée est dans leur esprit l'une des communautés importantes, mais une parmi d'autres, au travers desquelles l'homme se réalise et qui devrait à ce titre bénéficier d'une certaine autorité dans les domaines économiques et sociaux liés à la vie professionnelle. Ajoutons, pour terminer, qu'ils estiment également ce corporatisme social parfaitement compatible avec un régime politique démocratique.

L’idée de reconnaissance légale des corps intermédiaires sert également à fonder en droit les aspirations politiques des nationalités et à leur donner des garanties légales, dans la mesure évidemment où elles respectent la primauté du bien commun. Ces nationalités peuvent donc espérer jouir de certaines prérogatives politiques en régime fédéral pour assurer la conservation et l'épanouissement de leur culture. On comprend sans peine quelle est la portée de cette doctrine dans le cas canadien. Le respect

74. À au moins une occasion, cependant, les commissaires font explicitement référence au corporatisme, sans toutefois le nommer. En effet, après avoir affirmé qu'une certaine action directrice de l'économie est nécessaire, ils écrivent: «De deux choses l'une: ou l'État assume seul cette direction et instaure une économie de type socialiste, avec toutes les conséquences sociales et humaines d'une telle option; ou il organise la direction en assurant d'abord la collaboration des forces de production, tant des entrepreneurs que des travailleurs : c'est la conception chrétienne de l'ordre économique qui exige l'intervention de l'État, mais avant tout pour stimuler toutes les initiatives privées, les coordonner, et les ordonner au bien commun. Une telle collaboration des forces particulières et des pouvoirs publics peut, sans verser dans l'autoritarisme étatique, et en disciplinant les mécanismes naturels, assurer à une économie donnée son maximum d'efficacité et de stabilité.» Rapport Tremblay, op. cit., 3-I: 138. Nos italiques.

75. M. Chevrier, «La conception pluraliste... », loc. cit., 55-56. 
des différences nationales, qui justifie cette reconnaissance légale, n'est pour Arès qu'une application à un cas particulier du respect de la diversité de la vie sociale, des multiples formes qu'elle peut prendre. L'État central, quant à lui, doit être agent d'union, d'association entre les nationalités (et non pas d'unité, qui évoque l'uniformisation), et doit pour ce faire éviter de devenir force niveleuse au profit d'une des nations, «l'union des cœurs et des esprits ne pouvant s'obtenir par des moyens impérialistes ${ }^{76}$ ».

Dans un autre registre, une politique décentralisée conforme à l'esprit du fédéralisme social aurait pour avantage non négligeable de stimuler la vie civique. Pour le père Arès, rien ne contribuerait davantage à combattre le désintéressement des citoyens des pays démocratiques pour la politique (qu'on déplore déjà à l'époque) que de leur rendre, ainsi qu'aux communautés de base, le maximum de responsabilités politiques qu'ils sont en mesure d'assumer. La politique qu'il propose a pour but de combattre ce sentiment de dépossession face au pouvoir d'un État puissant et lointain, et par cette voie rendre la politique à sa fonction humanisante dont elle ne devrait jamais se détourner. Il est entendu pour Arès qu'une personne responsable de son destin et dont l'action a une réelle portée sur le plan politique, ne serait-ce qu'à l'échelle locale, est davantage intéressée au bien de toute la communauté et à la vie civique. Il en résulte pour cette personne un bien en soi, une réalisation plus complète de ses virtualités, sans parler des avantages pour la société elle-même.

Il ressort de cette analyse que l'ordre fédéraliste est l'ordre organique par excellence, dans lequel chaque membre a son autonomie, sa vie et sa fin propres, tout en étant ordonné en définitive au bien de l'ensemble, lui-même condition du bien de la personne:

L'ordre [que le fédéralisme] vise à établir, au contraire [de l'étatisme], est un ordre vivant et organique qui, rassemblant en un même tout, sans les détruire, les communautés et sociétés de base et intermédiaires, d'une part, laisse à chacune la possibilité de travailler au bien de ses membres, mais dans les limites de sa compétence et de sa fin spécifique, et d'autre part, demande à chacune sa collaboration en vue de réaliser la fin générale de l'organisme tout entier, à savoir la création d'un milieu de vie qui permette à l'homme de se développer conformément à sa nature ${ }^{77}$.

Ce foisonnement de vie à tous les échelons qu'encourage le fédéralisme social produit le milieu le plus riche et le plus dynamique pour la per- 
sonne, parce qu'il correspond à la nature profonde de l'homme et répond à ce qu'exige son plein épanouissement: autonomie, agir libre et responsable.

L'étude sur le fédéralisme préparée par Richard Arès pour le Rapport Tremblay a, à l'instar de la contribution de Minville, une portée qui dépasse les cadres de l'œuvre de son auteur pour embrasser toute la pensée sociale de la tradition intellectuelle canadienne-française à laquelle il se rattache. En effet, on ne peut trouver meilleure formule que celle de "fédéralisme social» pour décrire l'idéal temporel du nationalisme traditionaliste canadien-français et évoquer toutes ses références philosophiques, sociologiques et politiques.

\section{4 - CONTRE L'ÉTATISME CENTRALISATEUR}

Tout le Rapport Tremblay se veut une réfutation en règle de la politique centralisatrice et fortement étatiste menée par le gouvernement fédéral canadien dans la foulée de la crise des années 1930 et de la Deuxième Guerre mondiale. On peut ramener l'ensemble de cette réfutation à deux grandes critiques: en premier lieu, cette politique étatiste fait fi de l'esprit de prudence (au sens aristotélico-thomiste) qui est la règle d'or du bon gouvernement; en second lieu, elle constitue un amoindrissement pour la personne humaine et la détache de ses fins les plus hautes, de diverses manières. Cette seconde critique est la plus fondamentale.

\section{a) Un manque de «prudence »}

Le Rapport Tremblay reproche sévèrement aux partisans de l'étatisme centralisateur de donner à leurs idées et aux réformes qui en découlent le caractère d'une certitude scientifique et de concevoir leur application avec le fatalisme d'une exigence de l'histoire. Contre ces prétentions, les commissaires font valoir l'irréductibilité de la vie sociale, elle qui est si complexe, à quelque système abstrait que ce soit. "On voudrait donc transfigurer le monde, avec la simplicité et la rigueur des disciplines mathématiques" alors que dans le domaine de l'action humaine, les choses se passent souvent "d'une manière si furieusement irrationnelle ${ }^{78}$ ", écrit Charles de Koninck dans un passage qu'auraient endossé tous les commissaires. L'esprit humain, qui peut de grandes choses, est néanmoins incapable de se représenter le réel dans son intégralité sans le simplifier. Le politique avisé sait tenir compte des limites de ces représentations et 
se refuse à leur donner une valeur absolue dans l'exercice du gouvernement, se réservant toujours la liberté de prendre ses distances avec ces modèles rigides au gré des circonstances selon ce que lui dictent le sens commun, son jugement, la raison pratique. Cela n'est rien d'autre que la vertu de prudence. C'est à cette condition que la société, respectée dans sa diversité et sa complexité, peut produire tous ses fruits et est véritablement menée à sa fin.

C'est en abordant la question du keynésianisme et de son application au Canada durant l'après-guerre que les commissaires font valoir avec le plus de force ce manque d'esprit de prudence de la politique fédérale. L'analyse qu'ils en proposent est inspirée de celle que livre François-Albert Angers dans l'annexe $11 \mathrm{du}$ rapport $^{79}$. De prime abord, ils adoptent une position modérée face au keynésianisme comme doctrine économique: il s'agit possiblement là d'une "grande théorie économique ${ }^{80}$ ", et on ne s'oppose pas a priori à ce qu'on en tente certaines applications au Canada. Ce qui pose problème, c'est la manière avec laquelle on impose ces réformes au pays depuis la guerre. On soutient que le keynésianisme, si séduisant soit-il, n'en demeure pas moins une théorie au caractère plutôt abstrait qui n'a toujours pas fait ses preuves sur le terrain et dont on ne peut mesurer toutes les conséquences: «Une théorie, en effet, ne s'élabore pas sans beaucoup d'abstraction et d'hypothèses, et quand elle se prolonge dans des mesures politiques concrètes, elle est forcée de négliger trop de contingences pour qu'on puisse être sûr, a priori, de leur efficacité. Or, la théorie keynésienne est hautement abstraite et simplificatrice ${ }^{81}$.» Citant le célèbre historien de la pensée économique Joseph Schumpeter, le rapport rappelle la propension des économistes à manquer de prudence et à entraîner sans retenue les gouvernements dans des expériences hasardeuses, «[c]ar alors ils mettent en cause des valeurs qui ne sont pas d'ordre matériel et qui ne se laissent pas traiter avec la même rigueur mathématique que leurs données théoriques ${ }^{82}$ ».

Voilà précisément l'erreur des partisans des politiques keynésiennes au Canada, selon les commissaires: convaincus d'avoir trouvé une formule simple et passe-partout capable d'assurer définitivement la stabilité éco-

79. François-Albert Angers, La centralisation et les relations fédérales-provinciales, annexe $11 \mathrm{du}$ Rapport Tremblay, 163-238. La critique du keynésianisme qu'on trouve dans cette annexe d'Angers est toutefois un peu plus tranchée que celle du rapport.

80. Rapport Tremblay, op. cit., $2: 297$.

81. Ibid., 298.

82. Ibid., 299. 
nomique du pays, ils s'efforcent de l'appliquer systématiquement, envers et contre tout, sans paraître se questionner sur ses effets imprévus, même au prix du fédéralisme traditionnel, de la protection des droits et libertés qu'il offre ainsi que des acquis les plus importants du Québec - sans compter les impacts sur la personne humaine et les conditions de son épanouissement. La vertu de prudence exige pourtant une certaine retenue: il est légitime de vouloir expérimenter de nouvelles théories économiques, mais ces théories doivent s'adapter à nos structures politiques et à notre conception de la vie, non l'inverse, nous disent les commissaires. Dans le même sens, Angers ajoute ce qui suit dans son mémoire :

[...] théoriciens et cliniciens se laissent à ce point séduire par une théorie qu'ils finissent par prendre un dessin idéalisé des choses pour la réalité. [...] Sur le plan politique, [cet esprit] a abouti, surtout au Canada, à un souverain mépris des valeurs politiques, tellement la satisfaction d'avoir trouvé enfin la clef de la stabilité économique dans des formules simples paraissait l'emporter sur toute autre considération ${ }^{83}$.

C'est ainsi qu'au Canada, depuis la Deuxième Guerre mondiale, l'antique vertu de prudence aurait selon le Rapport Tremblay été complètement déclassée par la conviction et les certitudes toutes scientifiques de l'ingénieur social imbu de keynésianisme. Le résultat prévisible de cette tendance ne peut être que l'éclipse de la politique et à terme l'amoindrissement de la personne humaine.

\section{b) Un amoindrissement de la personne}

Toute conception de l'ordre social et politique idéal repose en définitive sur une conception philosophique donnée de ce qu'est l'homme, des conditions de son bonheur et de sa réalisation. Pour les commissaires, la cause est entendue: l'étatisme centralisateur procède d'une conception tronquée de l'homme, dans l'ordre des moyens comme dans celui des fins, une conception qui le coupe de ses sommets et le détourne des véritables voies de son salut. Là résident les raisons les plus profondes du refus de cet étatisme centralisateur.

La conception de l'homme dont ce régime procède est tronquée en ceci, d'abord, que dans l'ordre des fins elle le réduit à sa vie matérielle. "L'ordre des valeurs est ainsi renversé, écrit Minville: le succès, c'est la conquête de l'argent; la prospérité, l'élévation du niveau de vie individuel

83. F.-A. Angers, La centralisation..., op. cit., 201-202. 
et collectif ; la civilisation, la puissance économique ${ }^{84}$.»C'est en quelque sorte le symptôme d'un abandon complet à cette civilisation matérialiste et techno-scientifique dont Minville craignait qu'elle engloutit la vieille civilisation chrétienne fondatrice de l'Occident. Les commissaires n'entendent pas laisser passer sans protester:

[...] dans cette aventure où les partisans de la centralisation veulent entraîner le peuple canadien et la province de Québec en particulier, se trouvent engagées des valeurs humaines que ne sauraient remplacer ni la stabilité économique, ni le plein emploi, ni la sécurité sociale, même à supposer que la centralisation permette d'atteindre totalement ces trois objectifs ${ }^{85}$.

Cette conception diminuée des fins de l'homme se répercute dans l'ordre des moyens. Dans cette dynamique centralisatrice et matérialiste, tout ce qu'on attend de l'État est qu'il réponde à la demande, qu'il assure le bien-être matériel des citoyens de la manière la plus efficace possible. L'efficacité technique, le rendement, sont en quelque sorte devenus l'unique barème de son action. Foin de la responsabilité première de l'homme face à son propre salut, de l'apport des communautés qui naissent spontanément de la pratique de la vie sociale, de la richesse qui naît de la diversité du réel, des conditions de la réalisation du bien commun: l'État central prend en charge la société civile au nom de ses intérêts matériels étroitement définis et dessert non plus des citoyens mais des numéros, une clientèle de producteurs et de consommateurs. C'est prendre les moyens pour les fins, pensent les commissaires: «Sans doute, [bien-être matériel] et [efficacité technique] sont nécessaires, mais comme moyens, comme instruments en vue d'une vie plus haute: la vie de l'esprit, laquelle est surtout conscience, liberté, responsabilité, initiative, auto-décision, auto-gouvernement ${ }^{86}$."

En effet, la loi naturelle exige bien davantage que le simple souci de l'efficacité technique et du bien-être matériel. Toute l'organisation de la vie humaine doit tenir compte de la nature libre de l'homme, sans quoi elle risque de le détourner de ses fins, ou du moins de compromettre son plein épanouissement. La politique n'y échappe pas, et doit en tenir compte. C'est pourquoi le Rapport Tremblay privilégie l'initiative privée chaque fois que cela est possible ${ }^{87}$. Cette approche, qui fait de l'action

\footnotetext{
84. Rapport Tremblay, op. cit., $2: 81$.

85. Ibid., 275.

86. Ibid., 276.

87. Par «initiative privée», nous entendons ici toute initiative non étatique, et non pas seulement l'action de l'entreprise privée. Cela inclut par exemple le rôle de l'Église dans le domaine de la santé et de l'éducation.
} 
indirecte de l'État plutôt que de son action directe la norme, est commune à toutes les recommandations du Rapport. Les intellectuels traditionalistes canadiens-français n'ignoraient aucunement les problèmes de leur société et les défis auxquels elle devait faire face dans l'avenir. Simplement, ils ont tenté d'y répondre par des moyens adaptés à leur conception de l'homme, donc en faisant appel à l'initiative personnelle chaque fois que cela était possible. Procéder autrement reviendrait à renier les "valeurs humaines d'ordre politique " pour se laisser séduire par les sirènes d'un économisme antihumaniste:

Le premier reproche, par conséquent, que l'on peut et doit faire à la thèse centraliste en général, c'est celui de contribuer à ruiner des valeurs humaines d'ordre politique, c'est celui de soumettre aveuglément la politique à l'économie et de l'entraîner dans une aventure qui ne peut tourner qu'à son détriment, et donc au détriment du bien-vivre de la population canadienne tout entière ${ }^{88}$.

L’État, en régressant du "gouvernement des hommes » à l' «administration des choses", perd son caractère politique, qui est de conduire selon la vertu de prudence la société, et par-delà la personne humaine, à leur fin. À terme, c'est le spectre du totalitarisme qui guette les sociétés qui s'engagent dans cette voie, préviennent les commissaires non sans quelque surenchère verbale: on craint l'avènement d'un État tutélaire et omnipotent réglant dans le menu détail la vie humaine, qui ce faisant rendrait anémique la société civile et atrophierait le sens des responsabilités et d'indépendance de la personne ${ }^{89}$.

L'étatisme centralisateur met en péril le dynamisme même de la société civile, toutes ces petites sociétés naturelles qui se forment spontanément, qui font la richesse de la vie sociale et sur lesquelles est édifié l'ordre fédéraliste - tel du moins qu'il est conçu dans le Rapport Tremblay. Parmi ces communautés menacées par la centralisation, les commissaires se soucient tout particulièrement du sort des nations minoritaires comme le Canada français, la nation étant le milieu de vie culturel par excellence. La centralisation ne peut selon eux aboutir qu'à l'uniformisation, au laminage de la minorité canadienne-française en la privant de son autonomie politique, autonomie qui lui est nécessaire pour établir un régime social et économique conforme à sa conception du monde et à sa tradition. On comprend bien que les valeurs nationales, si importantes pour la personne car elles lui fournissent les cadres de sa pensée, pâtiront de ce désaccord 
avec les lois régissant le milieu. C'est en définitive la personne qui en fera les frais et en sortira diminuée.

\section{5 - LE CANADA ET LA CONFÉDÉRATION}

Quelle vision du Canada les commissaires défendent-ils, d'après leur conception générale de la politique? Il faut d'abord savoir que pour eux, l'esprit général de ce qu'ils appellent le «compromis» de 1867 offre tout ce qu'il faut pour assurer au Canada une vie politique harmonieuse et ordonnée, l'union entre les deux peuples fondateurs dans le respect de leurs différences. L'année 1867 représente en fait l'accomplissement de la lutte des Canadiens français pour la reconnaissance de leur liberté politique $^{90}$. À la suite d'une longue tradition, et notamment des thèses groulxistes, leur mot d'ordre est donc: «retour à la constitution ».

La grande qualité de l'Acte de l'Amérique du Nord britannique est précisément, aux yeux des commissaires, d'être une œuvre politique - un compromis entre les divers participants à la Confédération destiné à assurer le bien commun de l'ensemble, tout en tenant compte des circonstances particulières. Les Pères de la Confédération, en plus d'avoir eu l'audace de voir grand pour les colonies de l'Amérique du Nord britannique, ont aussi fait preuve à la fois de souplesse et d'originalité en combinant de manière inédite la tradition parlementaire britannique et le fédéralisme américain - pour tenir compte des différences régionales, surtout du besoin du Canada français de jouir d'une certaine autonomie politique pour assurer son épanouissement. Au terme de difficiles négociations, ils ont opté pour cette formule d'union dans le respect des différences bien qu'elle rendît plus complexe la vie politique du jeune Canada, ce qui témoigne aux yeux des commissaires de cet esprit soucieux d'ordonner la politique aux meilleurs intérêts de l'homme ${ }^{91}$.

Pour le père Arès, qui reprend la matière de ses recherches sur le sujet ${ }^{92}$, il est clair que la Confédération est "essentiellement» le fruit du travail des Canadiens eux-mêmes, et non d'abord une loi du parlement anglais. Celui-ci n'aurait fait que confirmer dans l'ensemble les choix des premiers. Le Rapport Tremblay se réclame donc de la théorie du pacte constitutionnel, qui fait son chemin depuis la fin du xix ${ }^{e}$ siècle chez les provincia-

90. Ibid., $2: 46$.

91. Ibid., $1: 34$.

92. Richard Arès, La Confédération: pacte ou loi? (Montréal, Éditions de l'Action nationale, [1950]), 76 p. ; Dossier sur le pacte fédératif (Montréal, [s.é.], 1941), 102 p. 
listes canadiens. Selon la version canadienne-française de cette théorie, la Confédération serait le fruit d'un double pacte: d'une part, d'un pacte entre les deux principaux groupes ethniques qui forment le Canada, Canadiens anglais et Canadiens français; d'autre part, d'un pacte entre les représentants des trois colonies qui allaient donner naissance aux quatre premières provinces du pays. L’entente entre les deux nationalités «visait à donner à chacune d'elles un statut officiel dans la Confédération, en même temps qu'un traitement égal», et faisait d'eux des "partenaires ${ }^{93}$ ". Aux yeux des commissaires, ce pacte qui a fondé le Canada prend valeur d'entente engageant moralement non seulement les Canadiens de 1867, mais aussi les générations futures.

Deux grands principes fondent le système politique canadien, nous dit-on dans le rapport: le parlementarisme et le fédéralisme. Le parlementarisme est basé sur la suprématie du parlement et fait reposer l'essentiel de la vie politique du pays sur le jugement des parlementaires, éclairé par la tradition législative, les usages, les conventions qui s'élaborent au fil du temps. C'est la prudence politique incarnée. Cependant, cette dynamique tend à la centralisation et présente un risque pour le fédéralisme. Celui-ci, en effet, est basé sur la primauté de la constitution et de l'organe censé en donner l'interprétation, le tribunal. Il limite en outre le pouvoir du parlement en répartissant ses pouvoirs entre deux ordres de gouvernement ${ }^{94}$. La tension entre ces deux pôles a marqué la vie politique canadienne depuis lors et donné lieu à des interprétations très divergentes de la Confédération, selon qu'on privilégie le pôle parlementaire ou le pôle fédéraliste. Sans surprise, les commissaires, abordant la constitution principalement dans la perspective du nationalisme canadien-français et de ses intérêts, donne la primauté à son caractère fédéraliste: "[L’Acte de 1867] a pour but principal, non pas de doter les différentes provinces d'institutions parlementaires qu'elles possédaient déjà, mais bien de les unir sur une base fédérative, et [...] en conséquence il doit être considéré comme un statut essentiellement fédératif ${ }^{95}$. » Les Lords du Comité judiciaire du Conseil privé de Londres, loin d’avoir faussé le sens profond de la Confédération, n’auraient fait, selon les commissaires, que préserver l'esprit du compromis bâti lors des réunions de Charlottetown, de Québec et de Londres (1864 et 1866$)^{96}$.

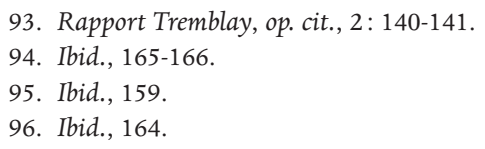


Le Rapport Tremblay reconnaît au départ sans difficulté l'existence d'un fort courant centralisateur parmi les Pères de la Confédération, un courant favorable à l'union législative des colonies plutôt qu'à leur fédération et qui est notamment à l'origine de ces pouvoirs spéciaux de contrôle des législatures provinciales qui ont été accordés au gouvernement central: pouvoir de désaveu, droit de nommer le lieutenant-gouverneur, pouvoir de réserve. Ils savent également que les promoteurs de la centralisation au Canada se réclament de son héritage - sur lequel ils basent leur conception de la Confédération. À cela, les commissaires répondent de deux manières. D’une part, ils citent à l'envi les discours des Pères les plus centralisateurs, dont John A. Macdonald, dans lesquels ceux-ci affirment que, bien que leur préférence va à l'union législative, l'étendue du pays et surtout la présence d'une forte minorité française dans l'ancien BasCanada rendent incontournable l'union fédérative. D'autre part, ils rappellent que Macdonald n'incarne pas à lui seul l'idée de la Confédération, que l'Acte de l'Amérique du Nord britannique est l'«expression d'un compromis entre des hommes dont les points de vue étaient sur certains sujets non seulement différents mais opposés ${ }^{97}$ ». En d'autres termes, les commissaires ne nient pas qu'un fort mouvement centralisateur ait marqué les pourparlers devant mener à la Confédération, mais prétendent que cette tendance a dû céder du terrain et composer avec des forces favorables à une plus grande autonomie des provinces, notamment chez les Canadiens français. Ce subtil équilibre entre les deux tendances constituerait le véritable esprit de la Confédération dont il faut continuer de s'inspirer, près de cent ans plus tard - et aussi d'après lequel il faut résoudre les difficultés posées par les ambiguittés du texte de la constitution, par exemple en matière de fiscalité.

Les commissaires ne se bornent toutefois pas à réclamer un Canada pleinement fédéraliste et décentralisé; ils portent une sorte de jugement moral sur la valeur respective des deux ordres de gouvernement, provincial et fédéral, conférant au gouvernement provincial une sorte de plusvalue sur le plan humain en raison de sa proximité avec ce qui donne le plus de prix à la vie humaine : éducation, vie sociale, municipalités. «Tout cela [les compétences provinciales] est en relation beaucoup plus étroite avec l'homme et avec les véritables fins de la société politique ${ }^{98}$ ", écrivent les commissaires dans le rapport, ajoutant que l'importance d'un pouvoir

97. Ibid., $2: 156$.

98. Ibid., $1: 40$. 
législatif ne se mesure pas à sa portée sur le plan géographique, mais bien à sa valeur sur le plan humain. Cela constitue même l'un des principaux critères servant à établir les recommandations fiscales de la commission, celle-ci tâchant d'attribuer aux deux ordres de gouvernement les types d'impôt les mieux adaptés à la nature de leurs fonctions et "à leur incidence sur les modes de vie, les institutions, la vie sociale ${ }^{99}$ ». Le gouvernement fédéral, quant à lui, est réduit à une sorte de service technique, de simple agent coordonnateur, dépendant sur le plan constitutionnel des provinces qui lui ont à l'origine donné l'existence et qui se trouvent être le véritable cour de la fédération canadienne. Il faut cependant bien admettre que tout Canadien anglais le moindrement nationaliste, même s'il se montre tout de même favorable à une certaine décentralisation, aurait beaucoup de difficulté à accepter qu'on vide ainsi de sa substance le gouvernement central, lien de tous les Canadiens et agent d'une vigoureuse politique nationale. Cette conception du fédéralisme canadien révèle un désintérêt assez évident à l'endroit du gouvernement fédéral, typique du nationalisme groulxiste.

«Les hommes de 1867 n'étaient peut-être ni des savants ni des grands penseurs, encore moins des prophètes, mais ils étaient le produit de traditions vivantes, qui leur donnaient une claire vision des choses et un sens net de la hiérarchie des valeurs ${ }^{100}$ ", écrit Esdras Minville dans le rapport, avant d'inviter à suivre leurs traces et à adapter l'esprit de leur œuvre politique aux conditions de son temps. Bien que la poussée centralisatrice des années 1940 et 1950 ait quelque peu ébranlé leur confiance, les nationalistes canadiens-français qui ont rédigé le Rapport Tremblay croyaient néanmoins encore, à leur manière, au Canada et à un certain idéal institué en 1867. Cette confiance allait rapidement s'effriter au cours des années suivantes, même chez les fédéralistes les plus réfléchis et convaincus, comme le père Arès.

Face à la montée d'une civilisation matérialiste et techno-scientifique, fondement philosophique de l'État-providence, le Rapport Tremblay prend fermement parti pour une civilisation chrétienne et personnaliste renouvelée, qui ordonnerait les acquis des sciences et de la technique au 
bien de la personne humaine au sens chrétien, considérée comme un être libre et raisonnable ayant la responsabilité première de son salut. Sur cette base, le rapport prend la défense de la politique et appelle à sa réhabilitation. Cette conception de la politique reconnaît d'abord l'aptitude de l'homme à se gouverner lui-même, à humaniser la terre, à orienter sa vie par la raison et le jugement vers le bien commun et ultimement vers le bien spirituel de la personne, fins fondées en nature de toute vie humaine. Cependant, sensible aux limites de l'homme, elle invite également à la sobriété, à une certaine humilité face à la complexité des sociétés humaines et au respect des contingences. Cette diversité sociale est au fond le reflet de la libre activité et de la sociabilité naturelle de l'homme - de l'irréductible diversité humaine elle-même. À trop vouloir réduire cette complexité pour mieux contrôler la société, c'est en définitive le sens de l'autonomie et de la responsabilité de l'homme qu'on risque d'étouffer. On en vient ainsi à perdre de vue la finalité dernière pour avoir voulu mieux atteindre des fins matérielles intermédiaires (stabilité de l'économie, lutte contre le chômage, etc.). Le rapport prend ainsi le contre-pied d'une conception du gouvernement réduite à de simples fonctions administratives, à des tâches d'intendance, avec comme seul barème de son action l'efficacité technique, sans tenir aucun compte des fins de l'homme. En cela, il s'inscrit dans un débat qui dépasse largement les frontières canadiennes. En effet, partout en Occident, les gens qui sont sensibles à la tradition et qui se méfient de l'esprit rationaliste appliqué à la politique mettent en garde contre les ingénieurs sociaux et leur prétention à changer la société en lui imposant rigidement des solutions dérivées de modèles abstraits. Force est de conclure que le Rapport Tremblay est une pièce de première importance à déposer contre la thèse de l'apolitisme des intellectuels canadiensfrançais que défendait le politologue André J. Bélanger dans un célèbre ouvrage publié il y a une trentaine d'années ${ }^{101}$.

De cette conception chrétienne de l'homme découle en outre l'exigence d'un ordre social décentralisé, laissant la part belle au dynamisme propre de la société civile, un dynamisme cependant ordonné au bien commun. Richard Arès rend remarquablement l'esprit de cette organisation par la formule très évocatrice de "fédéralisme social». La nation, selon le Rapport Tremblay, est une de ces communautés naturelles les plus importantes pour l'homme. Culturelle de par sa nature, ses ramifications s'éten-

101. André J. Bélanger, L'apolitisme des idéologies québécoises: le grand tournant de 1934-1936 (Sainte-Foy, Les Presses de l’Université Laval, 1974), 392 p. 
dent jusqu'aux domaines économique et politique - ce qui en fait l'une des bases fondamentales de la revendication autonomiste du Québec. Cela dit, la nation n'est pas la fin de tout pour les commissaires: ce qu'elle lègue à la personne est un bien des plus précieux, nécessaire à sa pleine réalisation, mais elle ne se confond pas avec l'ensemble du bien humain et n'épuise aucunement les aspirations de l'homme. L'autonomie politique que revendique le Rapport Tremblay pour le Québec et la lutte contre l'étatisme centralisateur dont il se fait le champion sont beaucoup plus que le fruit d'une obsession nationaliste, comme en témoigne le passage du rapport où est évoquée la possibilité que la politique québécoise suive les traces du gouvernement fédéral et que soit édifié au niveau de la province un État-providence contrôlé par des Canadiens français. Les commissaires sont catégoriques : l'étatisme centralisateur n'est pas plus acceptable sur le plan provincial que sur le plan fédéral, car il diminuerait de la même manière la personne et la société civile. Lisons leur conclusion:

Car ce qui donne un sens et du prix à l'autonomie provinciale, ce n'est pas que telle fonction soit exercée par le gouvernement de la Province, mais qu'elle soit exercée dans tel esprit - le gouvernement de la Province étant lui-même considéré comme le plus apte à comprendre et à faire triompher cet esprit. [...] Une politique sociale d'inspiration chrétienne selon les formes traditionnelles est donc la seule qui justifie la Province de Québec d'exiger le respect de son autonomie ${ }^{102} \ldots$

Le bien de la personne exige un ordre social décentralisé, ici comme ailleurs; de ce point de vue, la part du nationalisme en politique consiste à indiquer dans les grandes lignes quelle figure concrète doit prendre cet ordre décentralisé d'un milieu à l'autre, non de s'emparer du pouvoir et de l'exercer pour lui-même, au seul profit de la nation, sans tenir compte des droits des autres nationalités et des exigences supérieures de l'homme. Ce faisant, ils montrent bien que, tout important le nationalisme soit-il à leurs yeux, il n'en constitue pas une fin en soi et demeure ordonné à des fins plus hautes.

En somme, c'est tout un projet de civilisation que propose le Rapport Tremblay, un projet de civilisation dont les sources et la portée vont bien au-delà des limites de la petite société à laquelle il est adressé, tout en y étant profondément enraciné. Si certaines recommandations plus techniques du rapport en matière d'éducation, d'institutions culturelles, de

102. Rapport Tremblay, op. cit., 3-I : 134. Il s'agit là en quelque sorte d'une critique anticipée de la Révolution tranquille... 
planification économique par exemple, de même que l'idée générale d'un nationalisme politique proactif, ont réussi bon gré mal gré à trouver leur chemin jusqu'à nous, la lame déferlante de la Révolution tranquille allait cependant engloutir l'essence de ce projet de civilisation. Ce qu'il en reste, c'est le testament de tout un courant de pensée, une certaine idée, plus traditionnelle, du Canada français et du Québec, et des questions fondamentales posées à notre société, une société si obsédée d’un passé jugé arriéré qu'elle plonge tête baissée vers le progrès et l'avant-gardisme à tout prix. Ces questions pourtant nous interpellent toujours aujourd'hui: Qu'est-ce que l'homme? Qu'est-ce qu'une bonne vie? Comment la politique peut-elle les servir? 